\title{
Further Convergence Results for the Weighted Galerkin Method of Numerical Solution of Cauchy-Type Singular Integral Equations
}

\author{
By N. I. Ioakimidis
}

\begin{abstract}
The convergence of the weighted (jalerkin method (based on Chebrsher or Jacobi polynomials) for the direct numerical solution of one-dimensional. real Cauchy-type singular integral equations of the first and of the second kind on a finite interval is proved under sufficiently weak continuity assumptions for the kernels and the right-side functions of the integral equations.
\end{abstract}

1. Introduction. The problem of convergence of the weighted Galerkin method for the direct numerical solution of one-dimensional, real Cauchy-type singular integral equations of the second kind with constant coefficients on a finite interval (called in the sequel simply singular integral equations) [3], [5], [6], [8], [9], [11], [14] will be considered again. The results obtained supplement previous relevant convergence results [9], [11], [14] and, particularly, those of Linz [11] for singular integral equations of the first kind only. The present convergence results make use of the uniform norm like the results of [9], [11] and unlike those of [3], [8], [14].

The convergence of the above method will be proved under sufficiently weak continuity assumptions for the kernels and the right-side functions of the integral equations and will be mainly based on the method used by Linz [11] after a slight modification of this method. This modification consists in basing the convergence results on analogous results for Fredholm integral equations of the second kind [1], [2] and not proceeding directly with the singular integral equations under consideration as in [11]. Although this approach, already used in [3], [6], [8], [9], [14], may not be the most direct, it surely is the most economical, yielding a proof of convergence with little effort and on the basis of the well-known theory for Fredholm integral equations of the second kind [1], [2].

2. The Integral Equation. The real singular integral equation to be considered has the form

$$
a \varphi(x)+\frac{b}{\pi} f_{-1}^{1} \frac{\varphi(t)}{t-x} d t+\int_{-1}^{1} k(x, t) \varphi(t) d t=f(x), \quad-1<x<1
$$

The constants $a$ and $b$ are given, as well as the kernel $k(x, t)$ and the right side $f(x)$, which are assumed to be continuous functions. The unknown function is $\varphi(x)$, and

Received August 31, 1981: revised March 1, 1982 and October 18, 1982.

1980 Mathematics Subject Classification. Primary 65R20; Secondary 45E05, 45L10. 
it is assumed to be possibly unbounded as $x \rightarrow \pm 1$. Then [10]

$$
\varphi(x)=w(x) g(x),
$$

where

$$
w(x)=(1-x)^{\alpha}(1+x)^{\beta}
$$

with

$$
-\tan \pi \alpha=\tan \pi \beta=b / a, \quad-1<\alpha, \beta<0 .
$$

The number

$$
\kappa=-(\alpha+\beta)=1
$$

is the index [7] of (2.1). Since $\kappa$ was assumed equal to $1,(2.1)$ does not possess a unique solution unless supplemented by an additional condition, e.g.,

$$
\int_{-1}^{1} \varphi(t) d t=0
$$

The cases when $\kappa=0, \kappa=-1$ or $\kappa=1$ and 0 is replaced by a constant $C$ in (2.6) are quite similar [3] and will not be considered here.

Because of (2.2), we can rewrite (2.1) and (2.6) as

$$
\begin{gathered}
a w(x) g(x)+\frac{b}{\pi} f_{-1}^{1} w(t) \frac{g(t)}{t-x} d t+\int_{-1}^{1} w(t) k(x, t) g(t) d t=f(x), \\
\quad-1<x<1, \\
\int_{-1}^{1} w(t) g(t) d t=0 .
\end{gathered}
$$

We will also use the Fredholm integral equation of the second kind equivalent to (2.7) and (2.8) [7], [9], [10]

$$
g(x)+\int_{-1}^{1} K(x, y) g(y) d y=F(x), \quad-1 \leqslant x \leqslant 1,
$$

where

$$
\begin{gathered}
K(x, y)=w(y)\left[a w^{*}(x) k(x, y)-\frac{b}{\pi} f_{-1}^{1} w^{*}(t) \frac{k(t, y)}{t-x} d t\right] \\
F(x)=a w^{*}(x) f(x)-\frac{b}{\pi} f_{-1}^{1} w^{*}(t) \frac{f(t)}{t-x} d t, \\
w^{*}(x)=1 / w(x)=(1-x)^{-\alpha}(1+x)^{-\beta}, \quad 0<-\alpha,-\beta<1 .
\end{gathered}
$$

Before proceeding in our analysis, we will assume that the known functions $k(x, t)$ and $f(x)$ are not only continuous functions (with respect to $x$ and $t$ on $[-1,1]$ ), but also that they possess continuous derivatives (at least with respect to $x$ ) of orders $p_{1}$ and $p_{2}$, respectively, on $[-1,1]$, which are Hölder-continuous [7] with indices $\mu_{1}$ and $\mu_{2}$, respectively, that is,

$$
k, f \in C, \quad(\partial / \partial x)^{p_{1}} k \in H^{\mu_{1}}, \quad f^{\left(p_{2}\right)} \in H^{\mu_{2}} .
$$

The values of $p_{1,2}$ and $\mu_{1,2}$ sufficient for the assurance of the convergence of the weighted Galerkin method for the solution of (2.7) and (2.8) will be specified below. 
3. Convergence Results. We consider first the weighted Galerkin method [3], [5] of the numerical solution of (2.7) and (2.8). The existence of a unique solution of the system of linear algebraic equations approximating (2.7) and (2.8) was proved (under sufficiently weak assumptions) in [3]. The convergence of this method was proved in [11], [9] in the special case of singular integral equations of the first kind.

We use the notation $\varphi_{i}(x)$ for the Jacobi polynomial $P_{i}^{(\alpha, \beta)}(x)$ of degree $i$ corresponding to $\alpha$ and $\beta$, defined in (2.4), and $\varphi_{i}^{*}(x)$ for the Jacobi polynomial $P_{i}^{(-\alpha,-\beta)}(x)$. We assume that the function $g(x)$ has an expansion of the form

$$
g(x)=\sum_{i=0}^{\infty} c_{i} \varphi_{i}(x)
$$

where, because of the orthogonality properties of $\varphi_{i}(x)$ with respect to $w(x)$ on $[-1,1]$,

$$
c_{i}=\theta_{i}^{-1} \int_{-1}^{1} w(t) g(t) \varphi_{i}(t) d t
$$

with [13, p. 68]

$$
\theta_{i}=\int_{-1}^{1} w(t) \varphi_{i}^{2}(t) d t=\frac{2^{\alpha+\beta+1}}{2 i+\alpha+\beta+1} \frac{\Gamma(i+\alpha+1) \Gamma(i+\beta+1)}{\Gamma(i+1) \Gamma(i+\alpha+\beta+1)},
$$

where $\Gamma(x)$ denotes the gamma function. Since

$$
\Gamma(i+\alpha) / \Gamma(i+\beta)=\Theta\left(i^{\alpha-\beta}\right),
$$

we conclude from (3.3) that

$$
\theta_{i}^{-1} \leqslant A_{1} i
$$

where $A_{1}$ is a positive constant. We will use in the sequel the symbols $A_{m}$ to denote constants independent of $i$, but, probably, dependent on the functions used, the variables $x$ and $t$, etc.

Now we assume that $g \in C^{p}[-1,1]$ and further that $g^{(p)} \in H^{\mu}[-1,1]$. Then from a corollary of Jackson's theorem [12, pp. 22-23] we conclude that there exists a polynomial $p_{i}(x)$ of degree less than or equal to $i$ such that

$$
\left\|g-p_{i}\right\|_{\infty} \leqslant A_{2} i^{-(p+\mu)},
$$

where the uniform norm $\|g\|_{\infty}$ of a continuous function $g(x)$ is used. Since

$$
\int_{-1}^{1} w(t) p_{i-1}(t) \varphi_{i}(t) d t=0
$$

because of the orthogonality properties of Jacobi polynomials, we find from (3.2)

$$
c_{i}=\theta_{i}^{-1} \int_{-1}^{1} w(t)\left[g(t)-p_{i-1}(t)\right] \varphi_{i}(t) d t
$$

where $p_{i-1}(t)$ denotes the polynomial of degree $\leqslant i-1$ of best uniform approximation to $g(t)$. We further obtain from (3.8):

$$
\left|c_{i}\right| \leqslant \theta_{i}^{-1}\left\|g-p_{i-1}\right\|_{\infty} \int_{-1}^{1} w(t)\left|\varphi_{i}(t)\right| d t .
$$

Taking into account that [13, p. 171]

$$
\int_{0}^{1}(1-t)^{\nu}\left|\varphi_{i}(t)\right| d t \sim i^{-1 / 2}, \quad 2 \nu>\alpha-3 / 2
$$


(the symbol $\sim$ being given the interpretation adopted in $[13$, p. 1]), we see (since (3.10) holds for $\nu=\alpha$ ) that

$$
\int_{-1}^{1} w(t)\left|\varphi_{i}(t)\right| d t \leqslant A_{3} i^{-1 / 2}
$$

On the basis of (3.5). (3.6) and (3.11). (3.9) can be written as

$$
\left.\left|c_{1}\right| \leqslant A_{4} i^{-(p+\mu} \quad 1 / 2\right) \text {. }
$$

Hence, the following lemma is proved:

Lemma 1. Let $g \in C^{p}[-1,1]$ and $g^{(p)} \in H^{\mu}[-1,1]$. Then the coefficients $c$; of the expansion of $g$ into a series of Jacobi polynomials, determined by (3.2), satisfy (3.12).

As was shown in [9], the application of the direct weighted Galerkin method (with respect to $\left.w^{*}(x)\right)$ to $(2.7)$ and (2.8) is completely equivalent to the application of the same method (but with respect to $w(x)$ ) to (2.9). Moreover, it is also clear that the application of this method to (2.7) and (2.8) yields an approximation $g_{n}(x)$ of $g(x)$ of the form [1], [2]. [3], [5], [9]

$$
g_{n}(x)=\sum_{i=0}^{n} \gamma_{i} \varphi_{i}(x),
$$

which is the closed-form solution of (2.7) and (2.8) if $k(x, t)$ and $f(x)$ are similarly approximated by the finite series:

$$
\begin{aligned}
k_{n}(x, t) & =\sum_{k=0}^{n-1} \delta_{k}(t) \varphi_{k}^{*}(x), \\
f_{n}(x) & =\sum_{k=0}^{n-1} \varepsilon_{k} \varphi_{k}^{*}(x),
\end{aligned}
$$

obtained by truncating the corresponding infinite series.

On the basis of (3.13) to (3.15), we see that (2.7) and (2.8) take the form

$$
\begin{gathered}
a w(x) g_{n}(x)+\frac{b}{\pi} f_{-1}^{1} w(t) \frac{g_{n}(t)}{t-x} d t+\int_{-1}^{1} w(t) k_{n}(x, t) g_{n}(t) d t=f_{n}(x), \\
\int_{-1}^{1} w(t) g_{n}(t) d t=0,
\end{gathered}
$$

whereas (2.9) to (2.11) can be written as

$$
g_{n}(x)+\int_{-1}^{1} K_{n}(x, y) g_{n}(y) d y=F_{n}(x), \quad-1 \leqslant x \leqslant 1,
$$

with

$$
\begin{aligned}
K_{n}(x, y) & =w(y)\left[a w^{*}(x) k_{n}(x, y)-\frac{b}{\pi} f_{-1}^{1} w^{*}(t) \frac{k_{n}(t, y)}{t-x} d t\right], \\
F_{n}(x) & =a w^{*}(x) f_{n}(x)-\frac{b}{\pi} f_{-1}^{1} w^{*}(t) \frac{f_{n}(t)}{t-x} d t
\end{aligned}
$$

by application of the regularization procedure [7], [10] to (3.16) and (3.17). 
From now on we will work only with the Fredholm integral equations (2.9) and (3.18) to prove the convergence of the direct weighted Galerkin method applied to (2.7) and (2.8). We assume, to begin with, that (2.7) and (2.8) possess a unique solution. Hence, the same is true with (2.9) and, moreover, with (3.16) and (3.17), as well as with (3.18), for sufficiently large values of $n$. A large amount of theoretical results for Fredholm integral equations of the second kind is available [1], [2]. We can use, for example, Theorems $4.3,4.4$ or 4.5 of [2, pp. 426-430]. Theorem 4.4, slightly modified and adapted to our notation, states that

THEOREM 1. Suppose that

$$
\left\|(I+K)^{-1}\right\|_{\infty}^{*}\left\|K-K_{n}\right\|_{\infty}^{*}<1 .
$$

Then

(3.22) $\left\|g-g_{n}\right\|_{\infty}$

$$
\leqslant \frac{\left\|(I+K)^{-1}\right\|_{\infty}^{*}}{1-\left\|(I+K)^{-1}\right\|_{\infty}^{*}\left\|K-K_{n}\right\|_{\infty}^{*}}\left[\left\|F-F_{n}\right\|_{\infty}+\|g\|_{\infty}\left\|K-K_{n}\right\|_{\infty}^{*}\right] .
$$

In this theorem uniform norms of operators are also used [2, p. 22].

From (3.22) it is evident that the convergence of $g_{n}(x)$ to $g(x)$ is assured if $\left\|F-F_{n}\right\|_{\infty} \rightarrow 0$ and $\left\|K-K_{n}\right\|_{\infty}^{*} \rightarrow 0$ for $n \rightarrow \infty$. Moreover, if $\left\|K-K_{n}\right\|_{\infty}^{*} \rightarrow 0$ for $n \rightarrow \infty,(3.21)$ is also fulfilled under the already made assumption that (2.7) and (2.8) and, equivalently, (2.9) possess a unique solution. Hence $(I+K)^{-1}$ exists.

We now recall that $[13$, p. 166$]$

$$
\left\|\varphi_{i}\right\|_{\infty} \sim i^{q}, \quad i \rightarrow \infty
$$

where

$$
q=\max \left(\alpha, \beta,-\frac{1}{2}\right)=\max (\alpha, \beta)
$$

because of (2.4) and (2.5). Hence, we also have

$$
\left\|\varphi_{i}^{*}\right\|_{\infty} \sim i^{q^{*}}, \quad i \rightarrow \infty, \quad q^{*}=\max (-\alpha,-\beta)=-\min (\alpha, \beta) .
$$

By taking now into account that [10]

$$
\begin{gathered}
a w(x) \varphi_{i}(x)+\frac{b}{\pi} f_{-1}^{1} w(t) \frac{\varphi_{i}(t)}{t-x} d t=\frac{b}{2 \sin \pi \alpha} \varphi_{i-1}^{*}(x) \\
a w^{*}(x) \varphi_{i}^{*}(x)-\frac{b}{\pi} f_{-1}^{1} w^{*}(t) \frac{\varphi_{i}^{*}(t)}{t-x} d t=-\frac{2 b}{\sin \pi \alpha} \varphi_{i+1}(x)
\end{gathered}
$$

and the restriction (2.5), we easily obtain from (3.14), (3.15), (3.19) and (3.20)

$$
\begin{aligned}
K_{n}(x, y) & =-\frac{2 b w(y)}{\sin \pi \alpha} \sum_{k=0}^{n-1} \delta_{k}(y) \varphi_{k+1}(x), \\
F_{n}(x) & =-\frac{2 b}{\sin \pi \alpha} \sum_{k=0}^{n-1} \varepsilon_{k} \varphi_{k+1}(x) .
\end{aligned}
$$

Therefore,

$$
K(x, y)-K_{n}(x, y)=-\frac{2 b w(y)}{\sin \pi \alpha} \sum_{k=n}^{\infty} \delta_{k}(y) \varphi_{k+1}(x)
$$




$$
F(x)-F_{n}(x)=-\frac{2 b}{\sin \pi \alpha} \sum_{k=n}^{\infty} \varepsilon_{k} \varphi_{k+1}(x),
$$

On the basis of Lemma 1 and (3.23), we see further that

$$
\begin{gathered}
\left\|F-F_{n}\right\|_{\infty} \leqslant A_{5} n^{q+3 / 2-\left(p_{2}+\mu_{2}\right)}, \\
\left\|K-K_{n}\right\|_{\infty}^{*} \leqslant A_{6} n^{q+3 / 2-\left(p_{1}+\mu_{1}\right)} .
\end{gathered}
$$

Then from Theorem 1 it follows that:

THEOREM 2. Suppose that (2.13) and (3.21) hold and

$$
\gamma=\min \left(p_{1}+\mu_{1}, p_{2}+\mu_{2}\right)-(q+3 / 2)>0 .
$$

Then

$$
\left\|g-g_{n}\right\|_{\infty} \leqslant A_{7} n^{-\gamma}
$$

for the direct weighted Galerkin method of numerical solution of singular integral equations.

This theorem assures the convergence of the method under the assumptions already made. Consider as an application the case when $\alpha=\beta=-\frac{1}{2}, \mu_{1}=\mu_{2}=1$, $p=\min \left(p_{1}, p_{2}\right)$. Then from (3.34) $\gamma=p$, and Theorem 3 of Linz [11] is derived again. An improved convergence rate was obtained in [9] in this particular case.

Consider now in more detail the same special case where $\alpha=\beta=-\frac{1}{2}$ (but independently of the values of $\mu_{1,2}$ ). Because of (3.14) and (3.15), we obtain

$$
\begin{aligned}
\left\|f-f_{n}\right\|_{\infty} \leqslant A_{8} n^{-\left(p_{2}+\mu_{2}\right)} \log n, & a=0, \\
\left\|k-k_{n}\right\|_{\infty}^{*} \leqslant A_{9} n^{-\left(p_{1}+\mu_{1}\right)} \log n, & a=0,
\end{aligned}
$$

as is clear from the results reported in [12, pp. 22-23, 61].

Then, by working exactly as in [4, p. 288], we conclude, on the basis of (2.10), (2.11), (3.19), (3.20) and (3.36), (3.37), that

$$
\begin{aligned}
\left\|F-F_{n}\right\|_{\infty} \leqslant A_{10} n^{-\left(p_{2}+\mu_{2}\right)+\varepsilon}, & a=0, \\
\left\|K-K_{n}\right\|_{\infty}^{*} \leqslant A_{11} n^{-\left(p_{1}+\mu_{1}\right)+\varepsilon}, & a=0,
\end{aligned}
$$

where $\varepsilon$ is an arbitrarily small positive constant. These results are stronger than (3.32) and (3.33), respectively, for the special case considered $(a=0)$. The same results lead also directly to:

TheOREM 3. Suppose that $a=0$ and

$$
\gamma=\min \left(p_{1}+\mu_{1}, p_{2}+\mu_{2}\right)-\varepsilon>0,
$$

where $\varepsilon$ is an arbitrarily small positive coristant. Then Theorem 2 holds true for this value of $\gamma$.

Unfortunately, it has not been possible up to now to generalize this result for singular integral equations of the second kind. From Theorems 2 and 3 it follows also that under the assumptions made in this section the direct weighted Galerkin method of numerical solution of singular integral equations converges if $k, f \in$ $H^{\mu}[-1,1](0<\mu \leqslant 1)$ (with respect to the $x$-variable) in the case of singular integral equations of the first kind, and at least if $(\partial / \partial x) k, f^{(1)} \in H^{\mu^{*}}[-1,1]\left(\frac{1}{2} \leqslant \mu^{*} \leqslant 1\right)$ in the case of singular integral equations of the second kind. 
Acknowledgement. The results reported here belong to a research project supported by the National Hellenic Research Foundation. The financial support of this Foundation is gratefully acknowledged. The author is also grateful to the referee for many valuable comments.

Chair of Mathematics B'

School of Engineering

University of Patras

P. O. Box 120

Patras, Greece

1. K. E. AtKinson, $A$ Survey of Numerical Methods for the Solution of Fredholm Integral Equations of the Second Kind, SIAM, Philadelphia, Pennsylvania, 1976.

2. C. T. H. BAKer, The Numerical Treatment of Integral Equations, Clarendon Press, Oxford, 1977.

3. A. V. DzhishKariani, "The solution of singular integral equations by approximate projection methods," U.S.S.R. Computational Math. and Math. Phys., v. 19. no. 5, 1979(1980), pp. 61-74. [English translation of: Ž. Vyčisl. Mat. i Mat. Fiz., v. 19, no. 5, 1979, pp. 1149-1161.]

4. D. Elliott \& D. F. PAGET, "On the convergence of a quadrature rule for evaluating certain Cauchy principal value integrals: an addendum." Numer. Math., v. 25, 1976, pp. 287-289.

5. F. ERdogan, G. D. Gupta \& T. S. Cook" "Numerical solution of singular integral equations," Mechanics of Fracture, v. 1: Methods of Analysis and Solutions of Crack Problems (G. C. Sih, ed.), Ch. 7 , pp. 368-425, Noordhoff, Leyden, The Netherlands, 1973.

6. J. A. Fromme \& M. A. Golberg, "Numerical solution of a class of integral equations arising in two-dimensional aerodynamics," in Solution Methods for Integral Equations (M. A. Golberg, ed.), Ch. 4, pp. 109-163, Plenum Press, New York, 1979.

7. F. D. Gakhov, Boundary Value Problems, Pergamon Press and Addison-Wesley, Oxford, 1966.

8. M. A. GolBerg, "Galerkin's method for operator equations with non-negative index-with application to Cauchy singular integral equations," J. Math. Anal. Appl. (To appear.)

9. N. I. IoAKImIDIS, "On the weighted Galerkin method of numerical solution of Cauchy type singular integral equations," SIAM J. Numer. Anal.. v. 18, 1981, pp. 1120-1127.

10. S. KRENK, "On quadrature formulas for singular integral equations of the first and the second kind," Quart. Appl. Math., v. 33, 1975, pp. 225-232.

11. P. LINZ, "An analysis of a method for solving singular integral equations," BIT, v. 17, 1977, pp. 329-337.

12. T. J. RivLin, An Introduction to the Approximation of Functions, Blaisdell, Waltham, Mass., 1969.

13. G. Szego, Orthogonal Polynomials, rev. ed., Amer. Math. Soc. Colloq. Publ., vol. 23, Amer. Math. Soc., Providence, R.I., 1959.

14. K. S. Thомas. "Galerkin methods for singular integral equations," Math. Comp., v. 36, 1981, pp. 193-205. 